\title{
Mechanical Design of A Modular Quadruped Robot - XDog
}

\author{
Huixiang Xie* \\ Department of Mechanical Engineering \\ National University of Defense Technology \\ Changsha, P.R. China \\ E-mail: xhx-nudt@hotmail.com \\ *Corresponding author \\ Jianzhong Shang \\ Department of Mechanical Engineering \\ National University of Defense Technology \\ Changsha, P.R. China \\ E-mail: shangjianzhong@nudt.edu.cn
}

\author{
Zhixiong Zhang \\ Department of Mechanical Engineering \\ National University of Defense Technology \\ Changsha, P.R. China \\ E-mail: zzxnudt2010@sina.com
}

\author{
Zirong Luo \\ Department of Mechanical Engineering \\ National University of Defense Technology \\ Changsha, P.R. China \\ E-mail: lzrstone@163.com
}

\begin{abstract}
This article presents the mechanical design of a modular quadruped robot, named XDog. The leg mechanism of XDog has coplanar hip joints with the leg apart from the body to increase the motion range of the hip joint. Gear pair is used to drive each joint to promise high motion accuracy and compact assembling. Based on the leg mechanism and driving type, only one actuator is mounted on the leg with others in the torso to reduce the mass of the leg. Moreover, the leg design is modularized in a bilateral way, making each leg is capable for left or right side, and for forward or backward direction of the body. The mechanical design of XDog robot has advantages in large range of joint motion, light-weight leg, compact and high-accuracy driving system, and modular leg mechanism, making this design practical for developing a quadruped robot platform.
\end{abstract}

Keywords- mechanical design; legged robot; quadruped robot; modular design; leg mechanism

\section{INTRODUCTION}

Quadruped robots have become increasingly popular in the robotic community due to their potential capabilities of performing highly dynamic tasks. After decades' development, various quadruped robots with different structures were designed and implemented for diverse tasks [1-3]. Despite the control system, the mechanical design of a quadruped robot determines its performances in strength, accuracy and reliability, significantly influencing the capabilities of the robot.

The mechanical designs of quadruped robots are mostly inspired by quadruped animals. In terms of leg style, two types of leg structure are commonly used: the creeping insect type and cursorial mammal type. The insect type is with the leg horizontally sticking out of the torso (like a spider), such as TITAN-VIII [4] robot and SILO4 [5] robot. Such quadruped design is advanced in wide range of support area to execute climbing tasks or navigating rough terrain, but requires relative large joint torques to support the body due to the lateral extended leg structure.
Compared to the insect type mechanism, the mammal type leg grows downwards in sagittal plane (like a horse), producing wider work space in this plane for fast running. In addition, when the legs are fully extended and close to the singularity, less power is needed to generate large support force for the body weight and payloads. Hence, mammal type quadruped robots are more practical and popular. For example, the LittleDog [6], TekkenII [7], Cheetah-cub [8] and Rush [9] are all with this kind of design. These robots can perform fast trotting even on rough terrain. However, they are too small and weak to carry heavy payload like battery for significant endurance, and highly dynamic tasks like recover from critical lateral push are difficult for such platforms to carry out. Other examples such as HyQ [10], KOLT [11], BigDog [12, 13] and LS3 [14] are big quadruped robots with mammal type legs. These robots have powerful joints to run towards natural environments while carrying heavy payloads, but too big for social applications (such as family service or entertainment) and difficult to handle for experimental research.

Another important design for quadruped robot is the driving type of the joint, which influences the motion accuracy and reliability of the robot. For instance, direct driving through gears has high motion accuracy but always with actuators swinging with the leg, such as AIBO [15] and AiDIN [16], causing relative large inertial that needs more power to compensate. Another commonly used driving type is utilizing pulley and belt, or roller and rope. Quadruped robots with this type include KOLT [11], LittleDog [6], Cheetah-cub [8] and so on. The advantage of such driving mechanism is to move the actuators up to the torso of the robot, making the leg weight lighter. However, the soft diving chain would reduce the driving accuracy and efficiency.

In this paper, we present a modular design of a mammal quadruped robot, called XDog, with the size similar to a medium dog. The purpose of building XDog robot is for tests of locomotion control algorithms for 


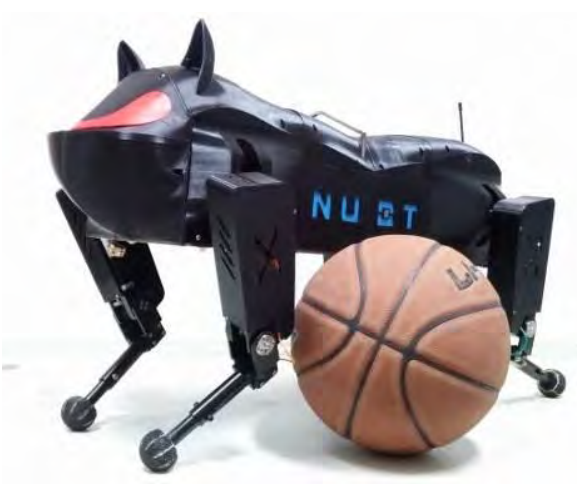

Figure 1. XDog robot compared with a basketball.

dynamic tasks, such as fast running, climbing slope, lateral push recover and so on. XDog is driven by DC motors and designed to have ability to carry payloads up to $4 \mathrm{~kg}$ compared to its net weight of $15 \mathrm{~kg}$. All of XDog's joints are driven through gears to promise high motion accuracy, and only one actuator is mounted on each leg (three actuated joints) with others in the torso to reduce the mass of the leg. Moreover, we modularize the leg design in a bilateral way, making each leg can be easily assembled either for the left or right side, and either with the knee joint pointing forward or backward.

\section{OVERVIEW OF XDOG ROBOT}

A photo of XDog robot is presented in Fig .1. XDog has three active degrees of freedom (DOF) in each leg: one hip abduction/adduction (a/a) joint, one hip flexion/extension (f/e) joint and one knee f/e joint. Each joint is directly actuated by a DC motor assembled with a planetary gearhead and an encoder. A compliant prismatic DOF with $10 \mathrm{~mm}$ of travel is set in the lower leg to absorb impacts with the ground.

$\mathrm{XDog}$ is designed to have medium body size to contain eight of the 12 actuators, all internal electronics (e.g. sensors, motor drivers and CPU, etc.) and power supply (i.e. batteries) within the main body. The physical dimensions of XDog are shown in Fig .2, in which the maximum robot height (hip to ground) is about $310 \mathrm{~mm}$ if the knee joints are fully extended. In order to reduce the weight of the robot, most of the parts of XDog are made from Aluminium Alloy except the driving gears and axes,

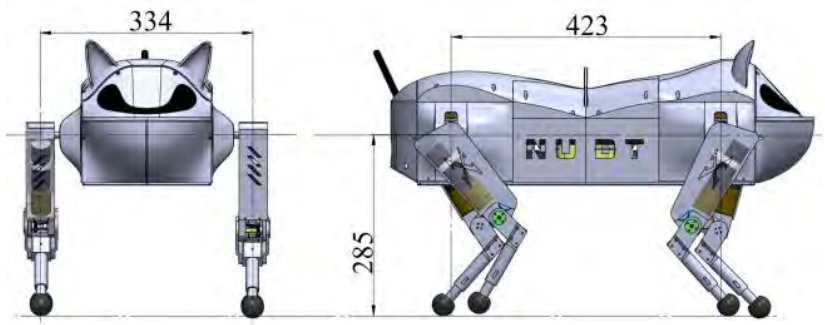

Figure 2. CAD model of XDog showing the dimensions (unit: $\mathrm{mm}$ ).

TABLE I. DIMENSION AND MASS PROPERTIES OF THE LEG

\begin{tabular}{|l|l|l|l|}
\hline Leg segment & Length $(\mathbf{m})$ & Mass $(\mathrm{kg})$ & Inertia $\left(\mathrm{kg} \cdot \mathbf{m}^{2}\right)$ \\
\hline Upper segment & 0.15 & 0.83 & 0.0067 \\
\hline Lower segment & 0.155 to 0.165 & 0.16 & 0.0011 \\
\hline Total & - & 0.99 & - \\
\hline
\end{tabular}

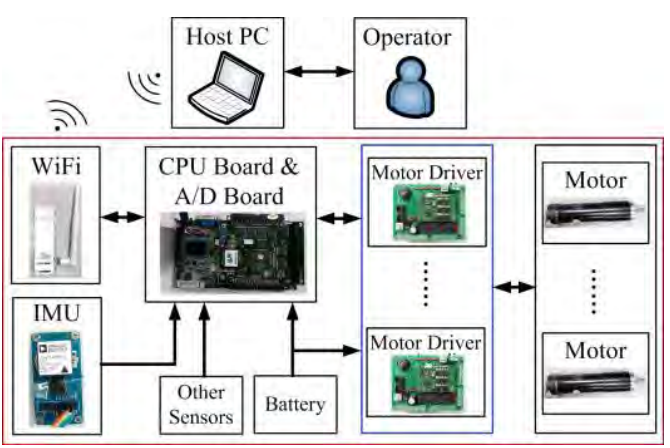

Figure 3. Overview of XDog's electrical system.

and the shell is printed by $3 \mathrm{D}$ printer with Polylactic Acid (PLA) material. As a result, XDog has a mass of $15 \mathrm{~kg}$ in total, while the four legs together weigh about $4 \mathrm{~kg}(27 \%$ of total mass) and all the 12 actuators weigh about $6 \mathrm{~kg}$ ( $40 \%$ of total mass). The detailed parameters of the leg are listed in Table I.

XDog has position sensors for each joint to sense the kinematics, an Inertial Measurement Unit to measure and calculate the robot velocities and orientations, and contact sensors in each leg to detect touch-down events. For current version, XDog does not have foot-force and visual sensors but is designed to easily update. The onboard computer consists of a CPU board and an A/D board, providing adequate USB, RS232 and I/O ports for sensors, motor divers and other embedded hardware. A host computer, which communicates with the onboard computer through a wireless internet, is used to set high level control commands and monitor the robot locomotion. XDog can be powered by an onboard battery or by external power supply. As part of the modular design, all the components of XDog's electrical system are available products from electronic market. See Fig .3 for an overview of XDog's electrical system.

\section{MECHANICAL DESIGN}

\section{A. Leg mechanism}

The leg mechanism is the most important part of the design of a quadruped robot. In order to have full dimensional maneuverability in 3D space, each leg should have at least three active joints, ordinarily with two perpendicular hip joints and one pitch knee joint. Fig .4 shows three possible leg mechanisms that can be used to

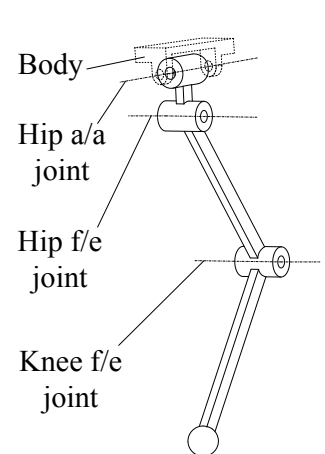

(a)

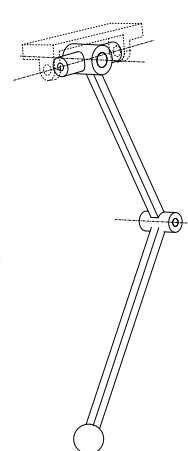

(b)

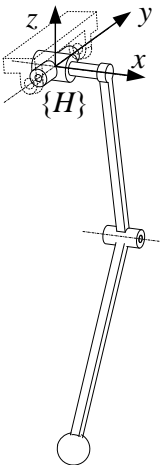

(c)
Figure 4. Three kinds of leg mechanism for quadruped robot: (a) separated hip a/a and f/e joints; (b) coplanar hip a/a and f/e joints; (c) coplanar hip joints with the leg apart from the body. 


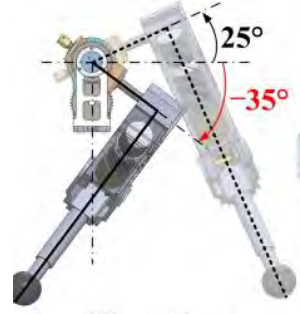

Hip a/a joint

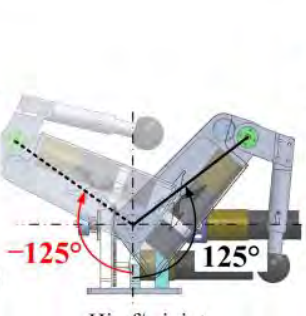

Hip f/e joint

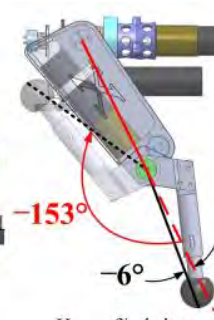

Knee f/e joint
Figure 5. Motion range of each joint

TABLE II. JOINT SPECIFICATIONS

\begin{tabular}{|l|l|l|l|}
\hline Joint & Motion range & Maximum torque & Maximum speed \\
\hline Hip a/a & $60^{\circ}$ & $7.09 \mathrm{Nm}$ & $98 \mathrm{rpm}$ \\
\hline Hip f/e & $250^{\circ}$ & $7.09 \mathrm{Nm}$ & $98 \mathrm{rpm}$ \\
\hline Knee f/e & $147^{\circ}$ & $7.09 \mathrm{Nm}$ & $98 \mathrm{rpm}$ \\
\hline
\end{tabular}

design a quadruped leg. The left one (Fig .4 (a)) is easy to implement since all joints are separated from each other, leaving enough space for actuator installation. However, when the leg swings in the lateral plane, the two actuators for the hip f/e and knee joints also swing with the leg, causing heavier swing leg. The leg mechanism of Fig .4 (b) has coplanar hip joints, so that the inertia of the leg can be reduced by moving the actuator of hip f/e joint up into the body, and the driving chains for the hip joints can be more compact. Nevertheless, due to the leg is straight under the body, the motion range of the hip f/e joint is restricted, ordinarily, less than $180^{\circ}$ to avoid collision with the body. To solve this problem, a simple way is to make a translation of the leg apart from the body, as shown in Fig .4 (c).

XDog is designed with the leg mechanism in Fig .4 (c), which brings large motion range for the hip f/e joint, promising the robot to totally squat down. The CAD model of XDog's leg is presented in Fig .5 to show the motion range of each joint and the joint specifications are listed in Table II. The large ranges of joint motion provide large range of feasible work space for the foot, and the large torque and fast speed of the joint allow the robot to perform highly dynamic tasks.

Compliance is significant to avoid mechanical damage causing by ground impacts, especially in fast running. $\mathrm{XDog}$ has a compliant prismatic joint in the lower leg, as shown in Fig .6. Two linear springs are used to absorb impacts and also store energy. Spring 1 is a strong one that

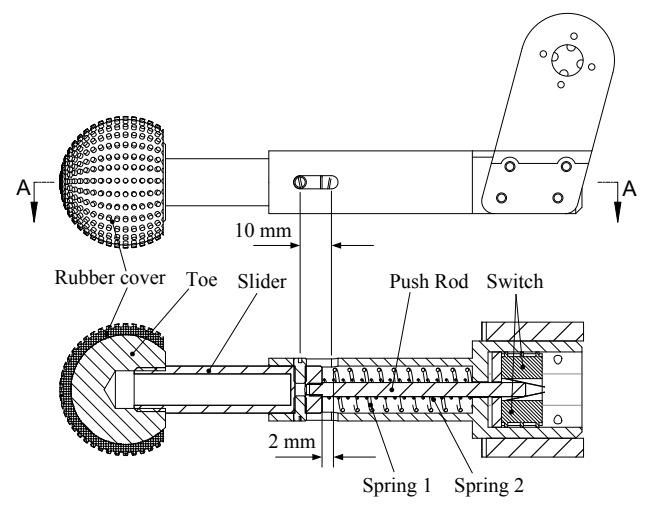

Figure 6. Design of the lower leg.

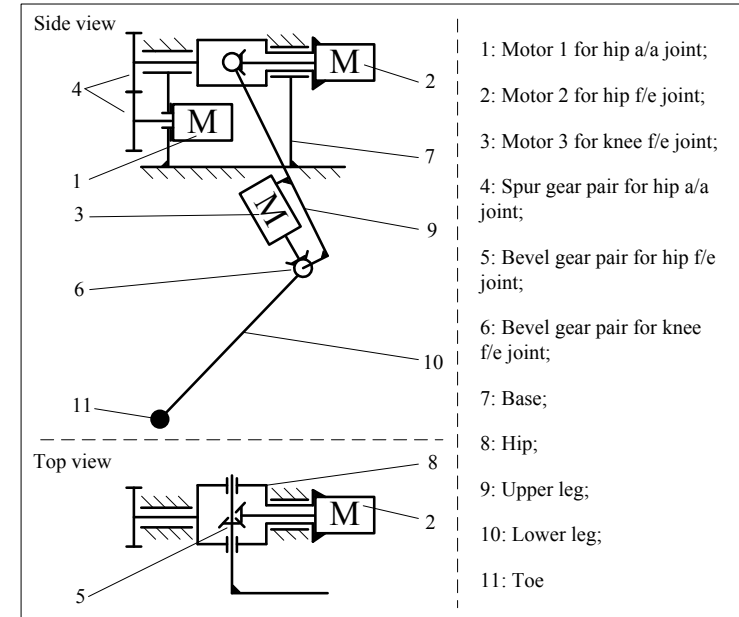

Figure 7. The driving system of XDog's leg.

has a small distance of $2 \mathrm{~mm}$ from the slider when the leg is in free length. This $2 \mathrm{~mm}$ distance, which is kept by a weak spring, Spring 2, is used to trigger the touch-down event through a push rod. Spring 1 works only after the switches are triggered by a small contact force. This design promise sensitive and reliable contact detection when the robot is walking. The ball-shape toe is covered by a layer of rubber to increase the frictional coefficient and also to dampen the ground impact. The toe can be easily replaced by new one embedded with force sensor.

\section{B. Driving System}

As we previously mentioned, gear transmission ensures high motion accuracy and large torque delivery. In this way, the actuator, here the motor has to be installed close to the joint. Fig .7 illustrates XDog's driving system, in which the knee f/e joint and the hip f/e joint are actuated through bevel gear pairs, and the hip a/a joint is actuated through a pair of spur gears. In Fig .7, only the knee joint motor is mounted in the swing part of the leg, with the others built in the body. Specially, Motor 2 is collinear with the axis of the hip a/a joint, so that it only rotates about its axis when the leg swings in lateral plane. This compact design of the driving system can reduce the inertial of the leg and save swinging space for motors inside the body.

\section{Leg Configuration}

XDog uses uniform leg design which is modularized in a bilateral way, as shown in Fig .8. By reversing the

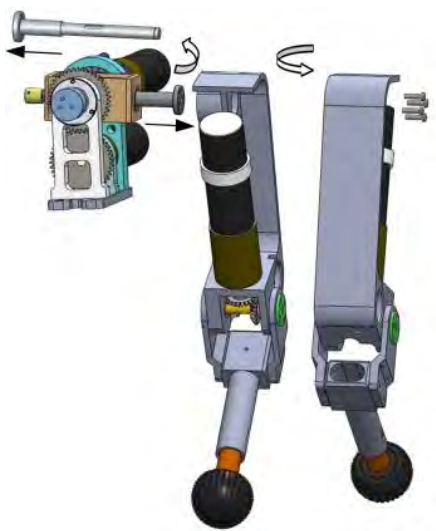

Figure 8. Modular design of XDog's leg. 


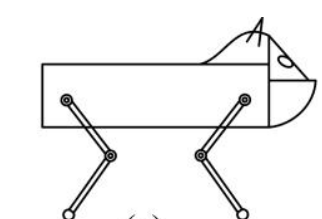

(a)

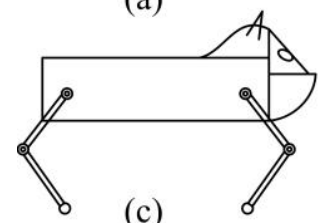

(c)

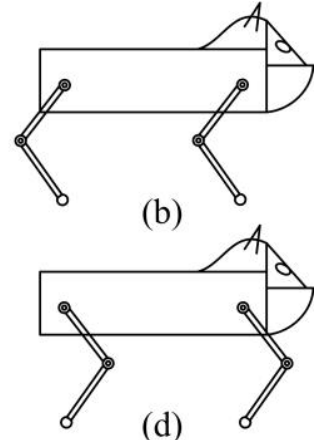

(d)

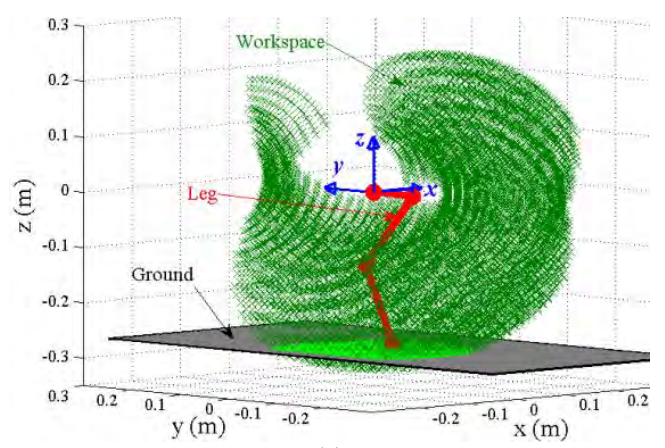

(a)

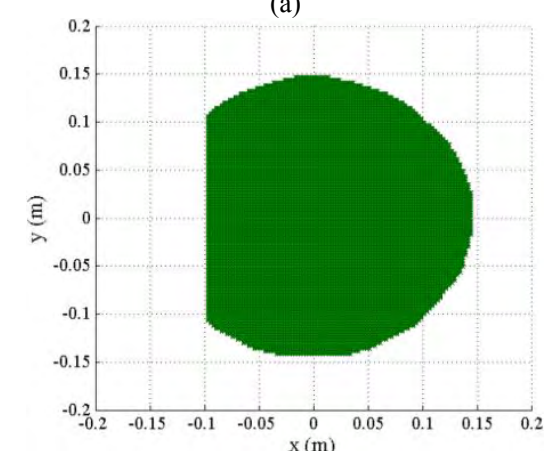

(b)
Figure 9. Leg configurations of a quadruped robot with knees pointing: (a) forward/backward; (b) backward/backward; (c) backward/forward; and (d) forward/forward.

rotation shaft of the hip f/e joint, the leg can be used either for the left or right side; by reversing the swing part of the leg, the knee joint can be chosen to point either forward or backward. The connection parts such as the hip and the upper leg are able to be assembled in both sides.

This design is useful for quadrupedal locomotion research when the robot needs to change its leg configuration, which can be defined according to the pointing direction of the knee joint. As shown in Fig .9, there are four different leg configurations for a quadruped robot: (a) the rear knees point forward and the front knees point backward, (b) all knees point backward, (c) the rear knees point backward and the front knees point forward, and (d) all knees point forward. It is conceivable that, with different leg configuration, the robot would have different performance for diverse tasks. Hence, it is convenient for XDog robot to change its configuration for locomotion research or various applications.

\section{Kinematics ANALysis OF Single LEG}

In order to prove the design of XDog's leg is capable for large work space, this section analyzes the kinematics of the single leg. According to the leg structure introduced in Fig .4 (c), the forward kinematics that maps the joint angles to the foot position with respect to the hip frame $\{H$ can be obtained using DH method.

As all joints go through their motion ranges, the work space of the foot is determined, as shown in Fig .10. In Fig .10 (a), the foot has large work space under and above the hip, ensuring wide range of footholds that can be selected for walking on irregular terrain. If a flat ground ( $0.285 \mathrm{~m}$ under the hip) is used to cut the work space, we can get an area of footholds as shown in Fig .10 (b), where the robot has maximum stride of $0.29 \mathrm{~m}$ in forward (y) direction and $0.24 \mathrm{~m}$ in lateral $(\mathrm{x})$ direction.

From Fig .10 we can see that, the foot has wider area of footholds on the ground if the robot stays a lower height, and this area becomes maximum when the hip height is about $0.2 \mathrm{~m}$. However, more bended leg needs larger joint torques for legged locomotion, resulting in inefficient walking. This analysis of the leg kinematics can be used to choose a better posture of the robot for locomotion control according to the requirements of certain tasks.

\section{DISCUSSION}

During the development of XDog robot, we notice that, beside the control methods, the actuator, power supply and Figure 10. Work space of the foot described in the hip frame: (a) space
formed by all reachable footholds compared to the leg; (b) area of footholds on a flat ground of $0.285 \mathrm{~m}$ under the hip.

materials significantly influence the robot performance and will change the direction of quadruped robot development.

The use of motor products from the market brings convenient access and ease of use for the actuation system. However, most available motor products are designed for applications that need continual rotation, which is not the case of a legged joint. As a result, the electric motor, which has low power-to-weight ratio with contradictory speed and torque capability, has to frequently reverse its direction of rotation, reducing its running efficiency. Besides, the weight of the motors makes up large percentage of the total mass of the robot (e.g. in XDog's case it is $40 \%$ ). Therefore, normal electric motor is not very appropriate for fast dynamic locomotion of legged robots, especially the big robots aimed at outdoor applications. As an alternate, a custom made motor with strong output and light weight would be able to improve the robot's actuation system, but will be more expensive. The power supply for electric actuators is battery. Although commonly used batteries (e.g. Lithium Polymer battery) can be built with large capacity, they also hold a significant weight of the robot $(13 \%$ in our case) if a long endurance is required.

So when building an electric actuated quadruped robot, we need to consider the power, weight and size of the motor, and endurance and stability of the power supply. The development of new technologies of motor and battery will provide light-weight motor with high power and enduring power supply with high power density. This will significantly change the exteriors of legged robots, resulting in lighter, compacter and stronger systems. By then, legged robot will be able to replace the wheeled fellows for space exploration.

Light-weight material and new manufacture method are partly used in XDog's development. For instance, by 
using 3D printing, ABS or PLA is used to manufacture the toe, shell and other non-structural components. However, the structural parts are still made from steel or aluminum alloy, holding a significant weight of the whole robot. Hence, if light-weight materials such as titanium alloy, carbon fiber or other new materials are used for body parts and leg segments, quadruped robot will have higher energy efficiency.

\section{CONCLUSIONS}

In this paper, we introduced details of the mechanical design of an electrical actuated quadruped robot named XDog, with focus on the design of the leg. The presented robot design has the following benefits:

(1) large work space of the foot, which profits from the translation of the leg apart from the body and is proved by kinematics analysis;

(2) light-weight legs ( $27 \%$ of total mass) based on high accuracy of joint motion by using gear driving; and

(3) modular leg that can be used for different robot configurations.

The mechanical design of XDog robot provides a good reference of building a quadruped robot. We are now developing the control system to test the performance of XDog robot, and will update it for various experiments of locomotion control. The points we discussed above also direct our future work.

\section{ACKNOWLEDGMENT}

This research work was supported by grants from the National High-Tech. Research and Development Program of China (No. 2011AA040801) and the National Natural Science Foundation of China (No. 51205400).

\section{REFERENCES}

[1] X. Zhou and S. Bi, A survey of bio-inspired compliant legged robot designs", Bioinspiration \& biomimetics, 2012, 7(4), pp. 1-20.

[2] S. Kajita and B. Espiau, Eegged robots", in Springer handbook of robotics. Springer, 2008, pp. 361-389.

[3] D. C. Kar, Đesign of statically stable walking robot: a review", Journal of Robotic Systems, 2003, 20(11), pp. 671-686.

[4] G. Endo, K. Arikawa and S. Hirose, An empirical comparison of a free dynamics simulator -Open Dynamics Engine" with TITAN-
VIII hardware torque/power measurements", 2011 IEEE International Conference on Robotics and Automation (ICRA), Shanghai, China, 2011, pp. 6065-6070.

[5] J. A. Galvez, J. Estremera and P. Gonzalez de Santos, A new legged-robot configuration for research in force distribution", Mechatronics, 2003, 13(8), pp. 907-932.

[6] M. P. Murphy, A. Saunders, C. Moreira, A. A. Rizzi and M. Raibert, The littledog robot", The International Journal of Robotics Research, 2011, 30(2), pp. 145-149.

[7] H. Kimura, Y. Fukuoka and A. H. Cohen, Adaptive dynamic walking of a quadruped robot on natural ground based on biological concepts", The International Journal of Robotics Research, 2007, 26(5), pp. 475-490.

[8] A. Spröwitz, A. Tuleu, M. Vespignani, M. Ajallooeian, E. Badri and A. J. Ijspeert, Fowards Dynamic Trot Gait Locomotion: Design, Control, and Experiments with Cheetah-cub, a Compliant Quadruped Robot", The International Journal of Robotics Research, 2013, 32(8), pp. 933-951.

[9] Z. Zhang and H. Kimura, Rush: a simple and autonomous quadruped running robot", Proceedings of the Institution of Mechanical Engineers, Part I: Journal of Systems and Control Engineering, 2009, 223(3), pp. 323-336.

[10] C. Semini, N. G. Tsagarakis, E. Guglielmino, M. Focchi, F. Cannella and D. G. Caldwell, - Đesign of HyQ-a hydraulically and electrically actuated quadruped robot", Proceedings of the Institution of Mechanical Engineers, Part I: Journal of Systems and Control Engineering, 2011, 225(6), pp. 831-849.

[11] J. G. Nichol, S. P. Singh, K. J. Waldron, L. R. Palmer and D. E Orin, System design of a quadrupedal galloping machine", The International Journal of Robotics Research, 2004, 23(10-11), pp. 1013-1027.

[12] M. Buehler, R. Playter and M. Raibert, Robots step outside”, Int Symp. Adaptive Motion of Animals and Machines (AMAM) Ilmenau, Germany, 2005, pp. 1-4.

[13] M. H. Raibert, K. Blankespoor, G. Nelson and R. Playter, Bigdog, the rough-terrain quadruped robot", Proceedings of the 17th World Congress, The International Federation of Automatic Control, Seoul, Korea, 2008, pp. 10823-10825.

[14] Boston Dynamics, http://www.bostondynamics.com" (Accessed on 22 Oct., 2014)

[15] M. Fujita and H. Kitano, Đevelopment of an autonomous quadruped robot for robot entertainment", Autonomous Robots, 1998, 5(1), pp. 7-18.

[16] I. M. Koo, T. H. Kang, G. L. Vo, T. D. Trong, Y. K. Song and H R. Choi, Biologically Inspired Control of Quadruped Walking Robot", International Journal of Control, Automation and Systems, $2009,7(4)$, pp. 577-584 